\title{
What Is the Association between an Anteriorly Tilted Pelvis and Trendelenburg Gait?
}

\author{
Yvonne Paul1, Mariette Swanepoel2, Terry J. Ellapen², Marco Barnard ${ }^{2}$, \\ Henriette V. Hammill², Reino W. Müller ${ }^{2}$, Juandré Williams²
}

${ }^{1}$ Department of Sport and Dental Therapy, Faculty of Health Science, Tshwane University of Technology, Pretoria, South Africa ${ }^{2}$ School of Human Movement Science (PhASRec), Faculty of Health Science, North-West University, Potchefstroom, South Africa Email: marcobarnard37@gmail.com

How to cite this paper: Paul, Y., Swanepoel, M., Ellapen, T.J., Barnard, M., Hammill, H.V., Müller, R.W. and Williams, J. (2018) What Is the Association between an Anteriorly Tilted Pelvis and Trendelenburg Gait? Open Journal of Orthopedics, 8, 464-475.

https://doi.org/10.4236/ojo.2018.812048

Received: October 5, 2018

Accepted: December 16, 2018

Published: December 19, 2018

Copyright () 2018 by authors and Scientific Research Publishing Inc. This work is licensed under the Creative Commons Attribution International License (CC BY 4.0).

http://creativecommons.org/licenses/by/4.0/

\begin{abstract}
Lower back pain (LBP) is a widespread, painful medical condition that has been plaguing society for many years. Present conservative rehabilitation focus is on lumbo-pelvic hip stability exercises in individual planes. However, a functional integrative rehabilitative approach addressing lumbo-pelvic misalignment in the sagittal (anteriorly tilted pelvis) and frontal (Trendelenburg gait) planes has not been presented. The aforementioned patho-biomechanical conditions and their management are often discussed estranged from each other rather than functionally integrated. This paper serves as a short communication which discusses the lumbo-pelvic anatomy, identifies the anatomical and biomechanical associations between the anteriorly tilted pelvic and Trendelenburg gait. Through an analysis of relevant literature, recommendations were made on the improvement of flexibility of the hip flexors, taut iliofemoral and pubofemoral ligaments to resolve the primary abnormal force-couple, with improved flexibility of the erector spinae and quadratus lumborum to resolve the secondary abnormal force-couple. In addition, improved flexibility of the hip flexors should coincide with closed-kinetic chain concentric strengthening of the ipsi-lateral hip abductors and contralateral external obliques. Patient education is also needed for self-re-alignment of the lower extremity to a neutral position and neutral foot stance. Biokineticists/exercise therapists should also review the patient's gait biomechanics to determine whether sartorius synergistic dominance is in play. In conclusion, the association between an anteriorly tilted pelvis and Trendelenburg gait, is in regard to taut anterior acetabulofemoral ligaments and femoral retroversion torsion angle that is both preceded and followed by the biomechanical influence of various anatomical structures. These anatomical and biomechanical factors must be evaluated by the biokineticists/exercise therapists before prescribing a rehabilitative programme to ensure successful rehabilitation of lumbo-pelvic hip
\end{abstract}


complex.

\section{Keywords}

Anteriorly Tilted Pelvis, Trendelenburg Gait, Interactive Functional Rehabilitation

\section{Introduction}

Lower back pain (LBP) is a common, painful and costly medical condition adversely impacting society, which can be traced backed to Imhotep (2800 BC) [1] [2]. LBP is also known as lumbo-pelvic pain [3] [4]. Lumbo-pelvic pain has been associated with intervertebral disc compression, misaligned innominate bones, sprain or taut ligaments and muscle asymmetry [5] [6]. Anteriorly tilted pelvic and Trendelenburg gait have been frequently identified as stressors of lumbo-pelvic pain [4] [7] [8]. In yesteryear, lumbo-pelvic pain was treated with bed rest, analgesics and surgery, however recently the rehabilitative focus has shifted to lumbo-pelvic stability exercise programmes, psychotherapy and refinement of ergonomic variables [2]. However, a functional interactive rehabilitative approach concerning the latter triad of maladies (misaligned innominate bones, sprain and taut ligaments as well as muscle symmetry) has not been adequately addressed, due to absence of an association between anteriorly tilted pelvis and Trendelenburg gait.

Exercise therapy students often ask the question: what is the association between an anteriorly tilted pelvis and Trendelenburg gait? Most prescribed literature used in the teaching of exercise therapy discusses the aforementioned conditions separately, creating the mind-set that these lumbo-pelvic hip conditions are estranged from each other [9] [10] [11] [12] [13]. However, these text books do discuss functional integrative rehabilitation as the ultimate goal of a functional exercise therapist, but none interactively identifies the commonalty of the aforementioned conditions and subsequently prescribes a functional interactive rehabilitation programme.

Subsequently comprehension and management of anteriorly tilted pelvis and Trendelenburg gait becomes a challenge to both the student and novice exercise therapist. The objective of this paper is to review the anatomical and biomechanical association between the anteriorly tilted pelvis and Trendelenburg gait in the sagittal plane and frontal plane respectively. The evidence of this clinical commentary will enhance the understanding of the LBP and its multi-planar contributors. Derived from this aforementioned knowledge, exercise therapists will be empowered to formulate a rehabilitative strategy to stabilize the lumbo-pelvic hip complex in a functional multi-planar dimension.

\section{Methods and Results}

The typical practices for systematic reviews, PRIMSA and its PICOS checklist for 
participants, interventions, comparisons, outcomes, and study designs were charted. The participants were records describing the relationship between Trendelenburg gait and anterior pelvic tilt; the intervention was not necessarily a therapeutic intervention but is interpreted as an exposure. The outcomes of interest were the relationship between Trendelenburg gait and anterior pelvic tilt. The exclusion criteria were 1) recording pertaining individually to Trendelenburg gait, 2) studies pertaining individually to anterior pelvic tilt.

A literature search was conducted utilizing peer-reviewed and professional journal publications, using the CROSSREF meta-database. CROSSREF includes the following databases: Pubmed, Medline, Science Direct, Ebscohost, Biomed, CINAHL, and Sabinet. The key search words were: relationship between Trendelenburg gait and anterior pelvic tilt. The screening eligibility of papers was performed in the following three steps: 1) title screen, 2) abstract screen, and 3) full-text screen. However, there were no results found describing the relationship between Trendelenburg gait and anterior pelvic tilt. Therefore, this short commentary theoretically describes the anatomical and kinesiological relationship between Trendelenburg gait and anterior pelvic tilt.

\section{Discussion}

\subsection{The Anatomy of the Lumbo-Pelvic Hip Complex}

The lumbo-pelvic hip complex is a coupling unit that transfers, absorbs, dissipates and amplifies force from the upper kinetic chain to the lower extremities and vice versa [10]. The lumbo-pelvic hip complex comprises of the acetabulofemoral joint, sacro-iliac joint (SIJ), pubic symphysis and the lumbosacral junction (Figure 1) [14]. The lumbosacral junction is an area where the entire weight of the trunk and upper extremities are transferred to the lumbo-pelvic complex [14]. The acetabulofemoral joint is a diarthrosis union of the femoral head and the acetabulum of the ilium, ischium and pubis [14]. The primary functions of
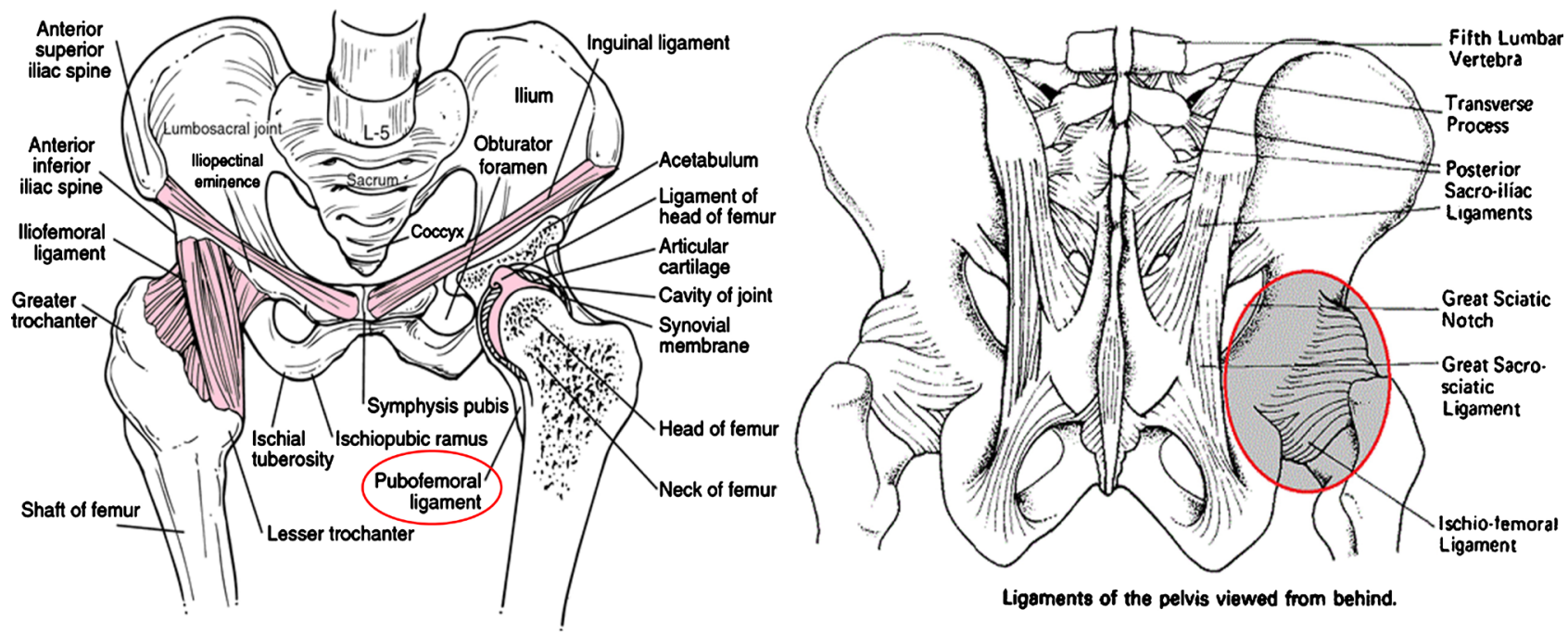

Figure 1. The lumbo-pelvic hip complex skeletal anatomy (http://www.medical-dictionary.com). 
this ball and socket joint are to provide a wide base of support for the lower extremities, abundant multi-planar range of motion and the kinetic link between the upper and lower extremities [13].

The muscles of interest in the sagittal plane include hip flexors and hip extensors. Hip flexors include iliopsoas (iliacus and psoas major), rectus femoris, sartorius and tensor fascia latae (TFL) (assisting hip flexion), while the antagonistic hip extensors are the gluteus maximus, biceps femoris (long head), semimembranosus, semitendinosus and adductor magnus (long head) (Figure 2) [13]. The abdominal muscles (rectus abdominus, external and internal obliques and transverse abdominus) provide lumbo-pelvic force closure in the sagittal, frontal and transverse planes acting as an abdominal brace [14]. Other lumbo-pelvic hip musculatures in the frontal and transverse planes include the abductors, external and internal obliques and transverse abdominus. Smaller transverse plane stabilizers include the external rotators (gluteus maximus, sartorius, piriformis, gemellus superior, gemellus inferior, obturator interior and exterior and quadrates

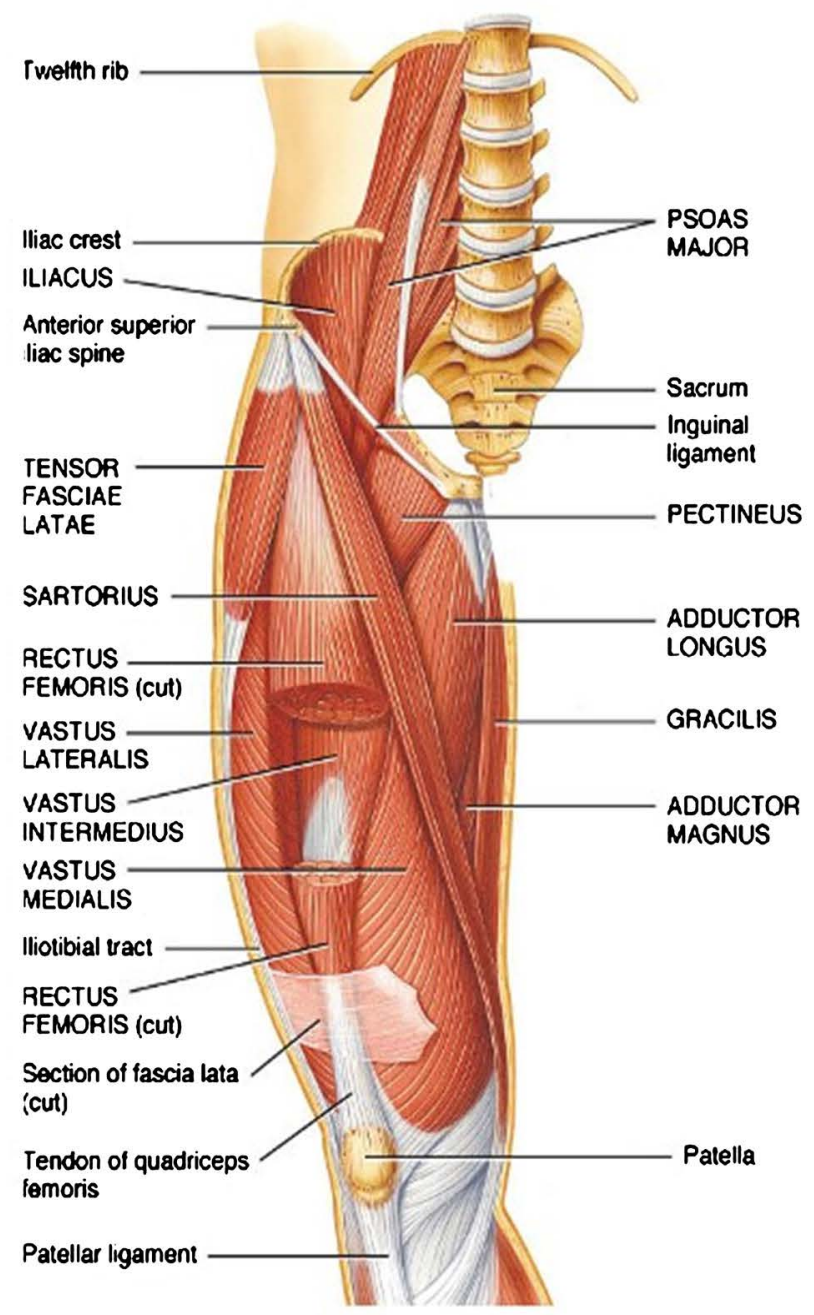

(a) Anterior suberficial view

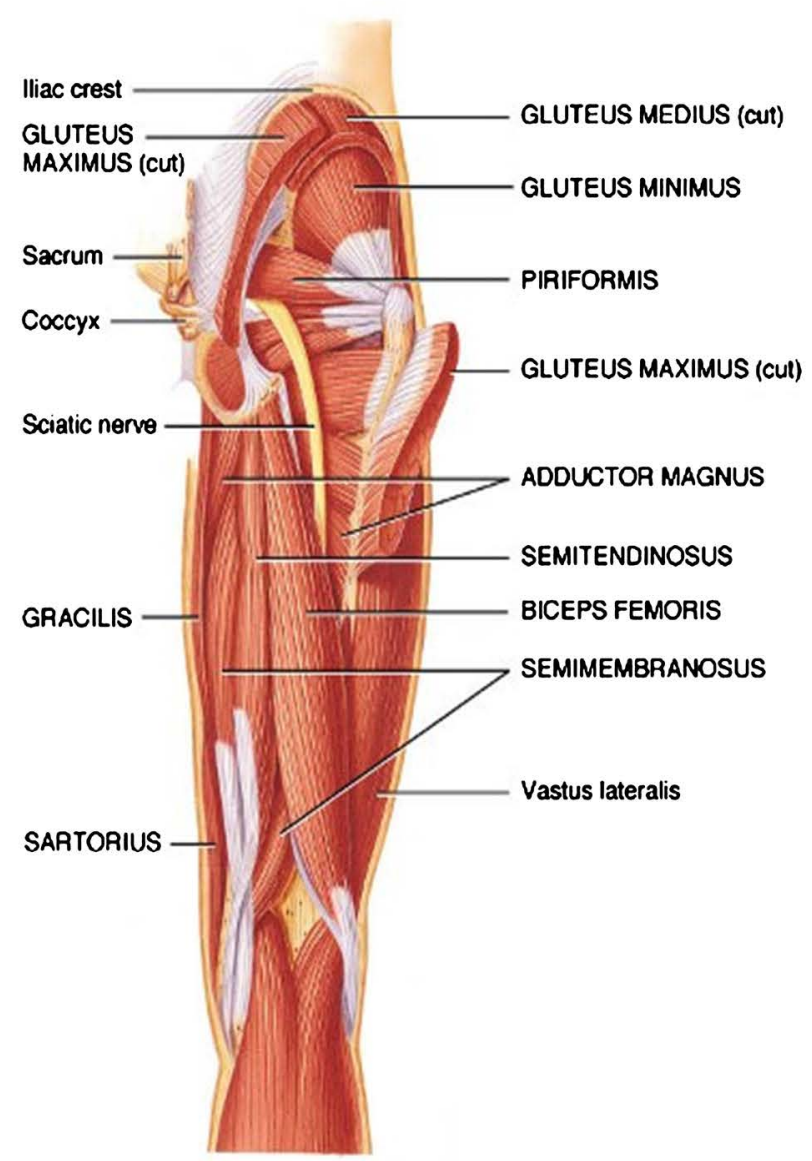

(b) Posterior suderficial view

Figure 2. The lumbo-pelvic hip complex musculature (http://www.boneandspine.com/hip-joint-anatomy.com). 
femoris) and internal rotators (gluteus medius (anterior fibres) and minimus and TFL) [14]. The anatomical and biomechanical significance of the functional interaction of these muscles in the various planes between an anteriorly tilted pelvis and Trendelenburg gait will be discussed later.

\subsection{Biomechanical Considerations of Short-Arc Pelvis-on-Femur Flexion (Anteriorly Tilted Pelvis)}

An anteriorly tilted pelvis is caused by hip flexor contractures, which by definition is a limitation to passive hip extension triggered by a lack of extensibility of the anterior acetabulofemoral ligaments and muscles [13] [15]. Individuals predisposed to an anteriorly tilted pelvis range from the elderly [16], administrators who sit for prolonged periods of time [17], individuals with abdominal ptosis [18], paraplegics' who are wheel-chair bound [19] to healthy strong athletes who asymmetrically strengthen their hip flexors more in relation to their hip extensors [20]. An anteriorly tilted pelvis (also biomechanically known as a short-arc pelvis-on-femur flexion), effects LBP [4], lumbar lordosis, limits rudimentary daily activities as well as sport performance [21]. Kendall et al. reported that the primary abnormal force-couple of a short arc pelvis-on-femur flexion is the iliopsoas and rectus femoris contractures that facilitates lengthening and weakening of the gluteus maximus and hamstrings [12]. Habitual short-arc pelvis-on-femur flexion precipitates taut iliofemoral and pubofemoral ligaments [15]. These ligaments are resistance to hip extension, but only the iliofemoral opposes external rotation and pubofemoral ligaments to abduction [13] [15]. The aforementioned patho-biomechanical implication indicates that short-arc pelvis-on-femur flexion contributes to the short-arc pelvis-on-femur adduction (Trendelenburg gait). Tautness of the pubofemoral ligament of the ipsi-lateral leg in the stance phase, resist hip abduction that will contribute to Trendelenburg gait (Figure 1).

The secondary abnormal force-couple of the short-arc pelvis-on-femur flexion is the lengthening and weakening of the rectus abdominus, internal and external obliques due to the contracture of the erector spinae (spinalis, longissimus and iliocostalis lumborum muscles and quadratus lumborum), as a consequence of the subsequent lumbar lordosis (Figure 3) [12]. The intra-vertebral musculature

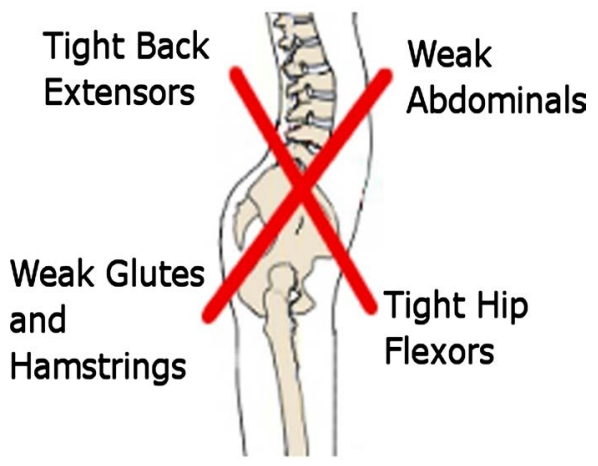

Figure 3. Short-arc pelvis-on-femur flexion [23]. 
and ligaments will not be discussed.

These abnormal force-couples of the short-arc pelvis-on-femur flexion in the sagittal plane radically influence the force closure of the lumbo-pelvic hip complex specifically due to the counter-nutation of the SIJ, which leads to muscular asymmetries in the frontal plane [21] [22]. It is important to remember that during short-arc pelvis-on-femur flexion, the ilium rotates anteriorly in the opposite direction of rotation of the sacral vertebrae [14]. Counter-nutation is the posterior rotation of the sacrum in relation to the anteriorly rotated ilium [14]. Thus counter-nutation affects the sacrospinous, sacrotuberous and iliolumbar ligaments to undergo a tensile force that weakens their static restraint, adversely reducing the joint congruency and stability in the sagittal plane (Figure 1). The weak and lengthened iliolumbar ligaments fail to assist the ipsi-lateral leg in stance phase to hold the contra-lateral hip horizontal that is in swing phase in the frontal plane, resulting in another biomechanical association of short-arc pelvis-on-femur flexion and short-arc pelvis-on-femur adduction.

\subsection{Recommendations}

It is recommended that exercise therapists should stretch the hip flexors and taut iliofemoral and pubofemoral ligaments to resolve the primary abnormal force-couple. This would reverse the anterior tilt of the ilium and the counter-nutated position of the SIJ. In addition, the erector spinae and quadratus lumborum should also be stretched to address the secondary abnormal force couple.

\subsection{Biomechanical Considerations of Short-Arc Pelvis-on-Femur Adduction (Trendelenburg Gait)}

Trendelenburg gait is characterized by hip abductor weakness that is unable to play a key role in the swing phase of walking and running [24]. During the stance phase of the ipsi-lateral leg, the contra-lateral hip (which is in the swing phase) is maintained at the horizontal height by the contraction of the ipsi-lateral hip abductors that is in a closed-kinetic chain movement (Figure 4). In closed-kinetic chain movements the proximal insertion (gluteus medius: between anterior and posterior gluteal lines of the posterior ilium and gluteus minimus: between the anterior and inferior gluteal lines) of the hip abductors move towards their distal attachments (greater trochanter of the femur) [25]. Therefore, drop in the contra-lateral hip height identifies weakness of the ipsi-lateral hip abductors of the stance leg [24].

Kendall et al. reports that muscles have dynamic interrelationships that expand across different planes of motion whose functional role change from primary agonists to synergists [12]. With that being said, the abdominal muscles (internal and external obliques and transverse abdominus) stabilize the lumbo-pelvic hip complex in the transverse plane by compression, acting as a corset, while they serve as synergists to the hip abductors in the frontal plane [12] [21]. During the swing phase of the gait cycle, the contra-lateral hip and its respective 


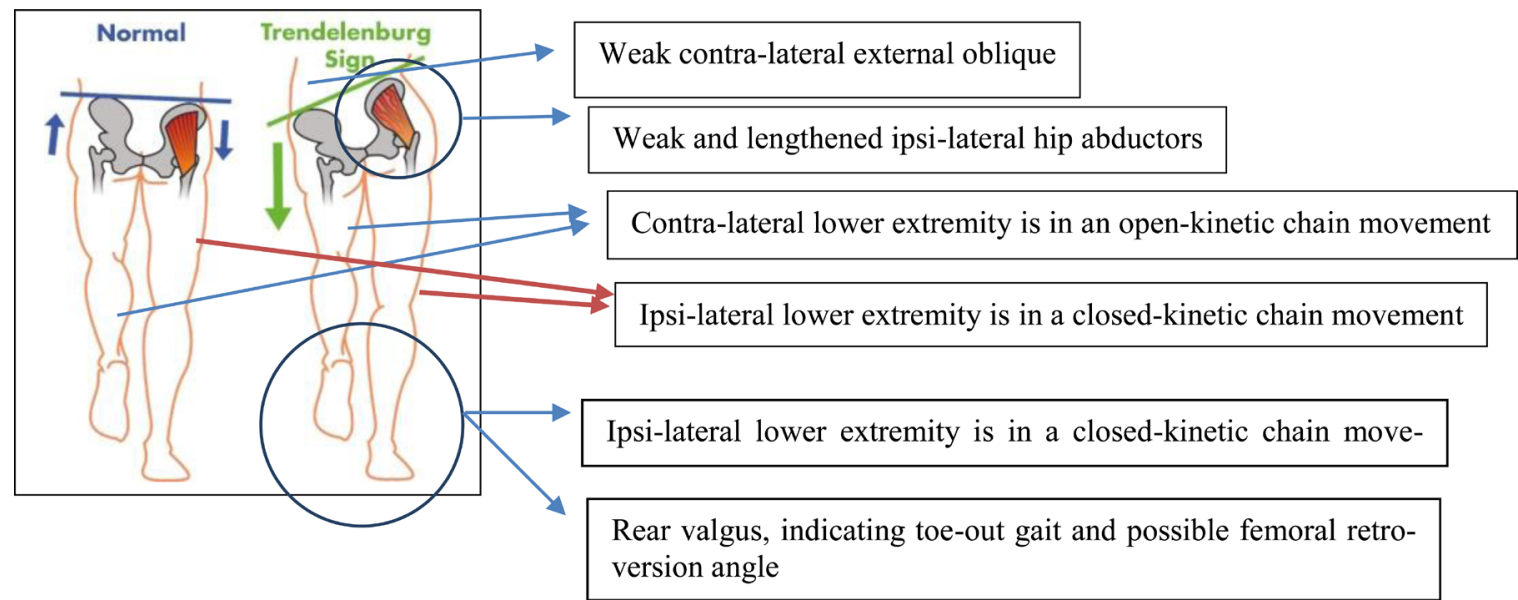

Figure 4. Short-arc pelvis-on-femur adduction (http://www.neuros.org).

lower extremity is in an open-kinetic chain movement, which necessitates the synergistic concentric contraction of the contra-lateral external oblique muscle to move its distal attachment towards it proximal site, in order to keep the entire hip in equilibrium [12] [21]. Failure to accomplish this, can result in a drop in the aforementioned contra-lateral hip [12] [21]. Paul et al. reports lengthening and subsequent weakness of the external obliques occurs because of anteriorly rotated pelvic in the sagittal plane, which affects its frontal plane synergistic functions [21].

Another biomechanical intrinsic factor that comes into play is the synergy of the TFL and sartorius to act as hip abductors, which creates the issue of synergistic dominance [14]. In an attempt to compensate for this weakened hip abductor torque deficit, the lumbo-pelvic compensates by increasing the contractility demand on the synergists TFL and sartorius to assist with hip abduction. TFL synergistic dominance affects the force-couple relationship of the tibiofemoral adductors and abductors leading to iliotibial band friction syndrome [26]. However, chronic synergistic dominance of the sartorius as a hip abductor in the frontal plane produces hypertrophy and hypertonicity that affects the functional capacity of the sartorius in all the planes. The stronger sartorius is also a hip flexor in the sagittal plane that increases the contracture of the hip flexors in the sagittal producing anteriorly tilted pelvis. This is another biomechanical association of short-arc pelvis-on-femur adduction in the frontal plane and short-arc pelvis-on-femur flexion in the sagittal plane.

\subsection{Recommendations}

It would be valuable to stretch the hip flexors and concentrically strengthen the ipsi-lateral hip abductors and contralateral external obliques with closed-kinetic chain exercises, further adjusting the hip's retroversion torsional angle by teaching the patient to re-align their lower extremity to a neutral position and neutral foot stance. Biokineticists/exercise therapists should also review the patient's gait biomechanics to determine whether sartorius synergistic dominance is in play. 


\subsection{Rehabilitative Exercise Program}

The following rehabilitative exercises are mere recommendations to increase stability of the lumbo-pelvic hip complex in all three planes (frontal, sagittal and transverse). It is advised that patient should first consult with a medical practitioner to confirm hip pathology and then consult a physiotherapist for myofascial release, before the biokineticist/exercise therapist begins a rehabilitative programme. The rehabilitative programme (Table 1) is an interactive functional programme attempting to rehabilitate both aforementioned lumbo-pelvic misaligned conditions in the frontal and sagittal planes. All stretches are static in

Table 1. Proposed rehabilitative exercise program.

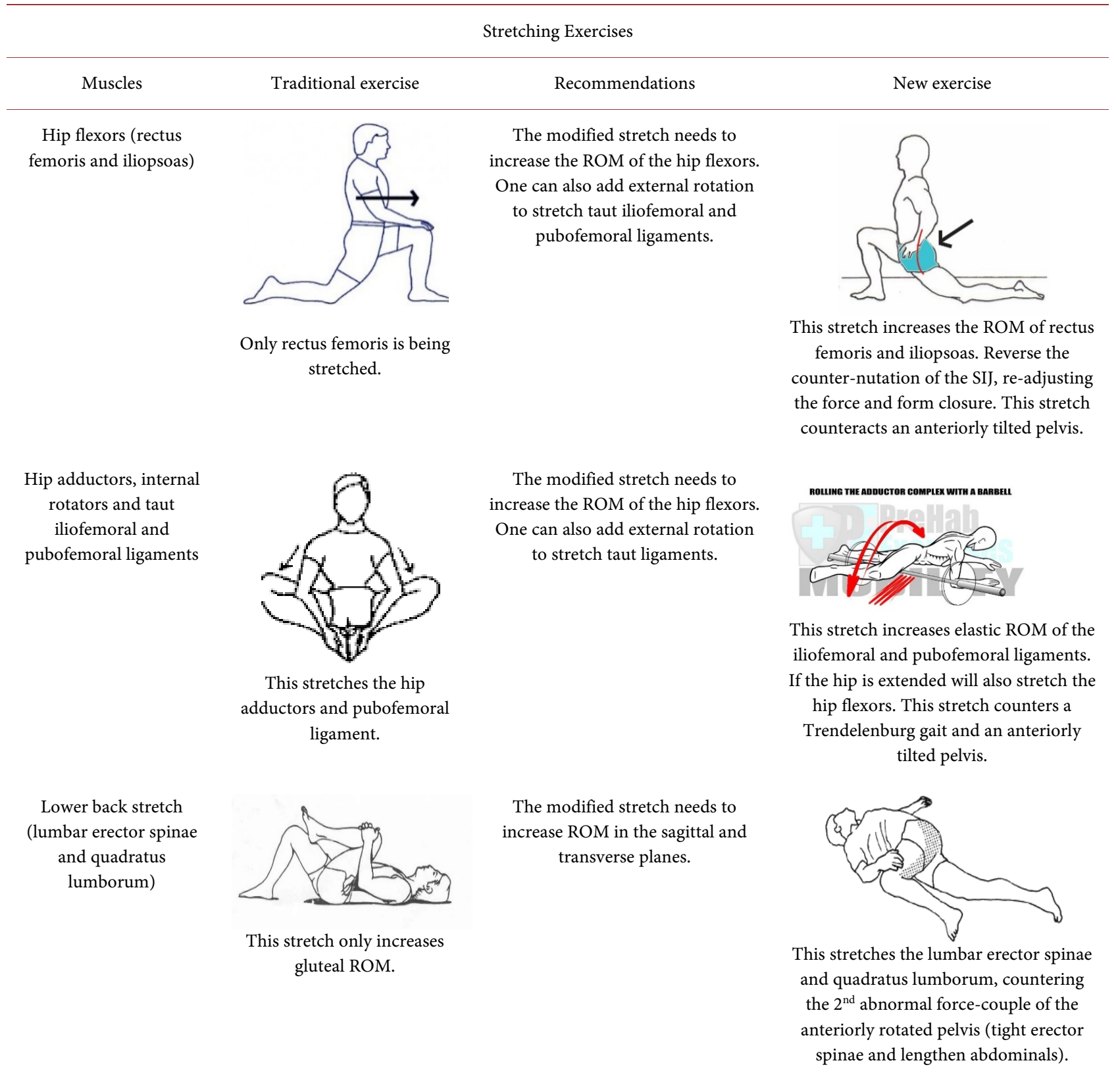




\section{Continued}

Strengthening Exercises

Mostly rectus
Contra-lateral external
obliques and ipsi-lateral
obliques
obliques internal
obliques transverse
abdominus
plane stabilization and no lumbo-pelvic force closure.

Hip abductors (gluteus medius and minimus)

Hip abductors (gluteus medius and minimus)

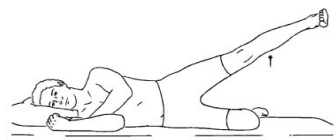

Frontal plane movement in an open-kinetic chain movement. However, hip abductors need to be strengthened in a closed-kinetic chain movement.
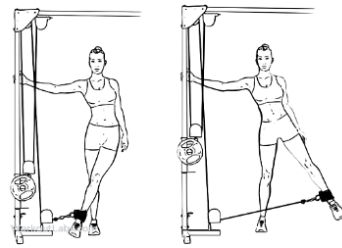

Frontal plane movement in an open-kinetic chain movement. However, hip abductors need to be strengthened in a closed-kinetic chain movement.
This exercise needs to strengthen hip abductors in a closed-kinetic chain movement to directly rehabilitate Trendelenburg gait.

This exercise needs to strengthen hip abductors in a closed-kinetic chain movement to directly rehabilitate Trendelenburg gait.
Multi-planar exercises

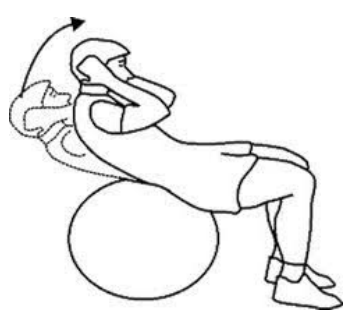

Patient has to enforce transverse and frontal plane stabilization that increases lumbo-pelvic force closure. This exercise counters an anteriorly tilted pelvis.

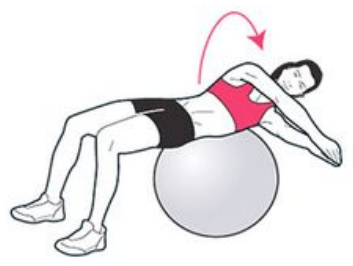

Patient has to enforce frontal and sagittal plane stabilization that increases lumbo-pelvic force closure. This exercise counters an anteriorly tilted pelvic.

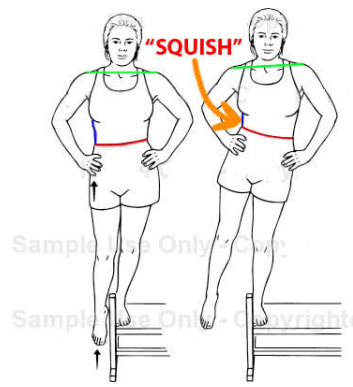

This exercise activates the muscles in a closed-kinetic chain movement, countering Trendelenburg gait.

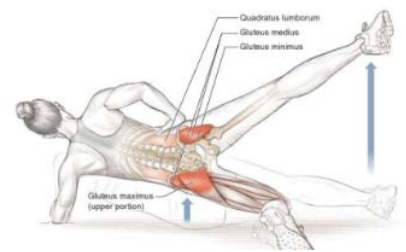

This exercise activates the targeted muscles in a closed-kinetic chain movement, countering Trendelenburg gait. The ipsi-lateral leg is in contact with ground, while the contra-lateral hip flexors are activated. 


\section{Continued}

Hip extensors (Gluteus maximus)

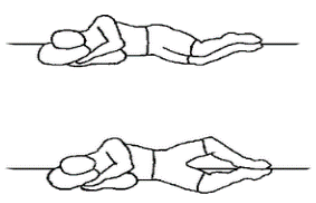

Only transverse plane muscle activation. No lumbo-pelvic force closure.

Modified plank

Multi-directional 3D-lunge

This exercise is a uni-planar exercise, movement in the sagittal plane. It has

lumbo-pelvic stabilization.
This exercise needs to strengthen hip abductors in a closed-kinetic chain movement to directly rehabilitate Trendelenburg gait.

Bi-planar movements, in the sagittal and transverse planes are needed. The exercise only enforces frontal plane stabilization. The multi-planar movement activates lumbo-pelvic force closure and stability. It counters both an anteriorly tilted pelvis and a Trendelenburg gait.

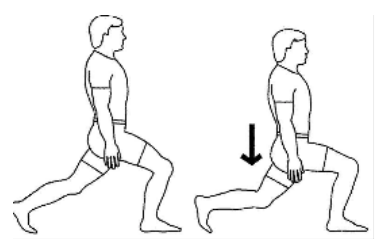

This lunge is a uni-planar movement.
Bi-planar movements, in the sagittal and transverse planes are needed. The exercise only enforces frontal plane stabilization. The multi-planar movement activates lumbo-pelvic force closure and stability. It counters both an anteriorly tilted pelvis and a Trendelenburg gait.

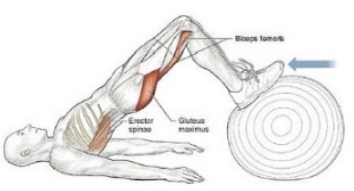

Patient has enforced transverse and frontal planes stabilization, thereby increasing lumbo-pelvic force closure and stability.
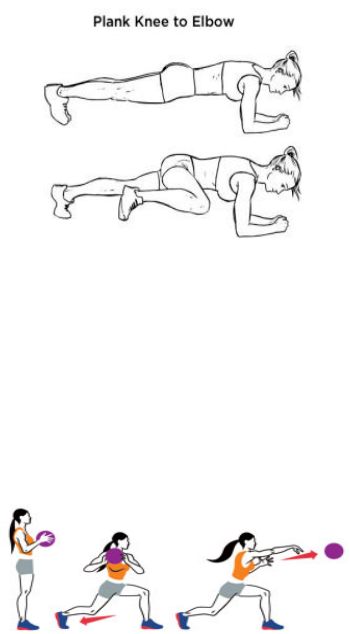

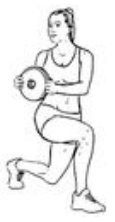

nature that should be maintained for 15 seconds, three repetitions each. All strengthening exercises focus on local muscle activation and should be completed as 20 repetitions of three sets. This rehabilitative programme should be completed every alternate day for three weeks. Thereafter, patients should be re-evaluated by a biokineticists/exercise therapist to determine quantitative progress.

\section{Conclusion}

The association between an anteriorly tilted pelvis and Trendelenburg gait, is in regard to taut anterior acetabulofemoral ligaments and femoral retroversion torsion angle. Slackened iliolumbar ligaments fail to synergistically support the ipsi-lateral hip abductors to hold the contra-lateral hip in equilibrium in the frontal plane, resulting in Trendelenburg gait. The iliolumbar ligaments became slackened due to the anteriorly tilted pelvic in the sagittal plane. Weakened, 
lengthened contra-lateral external obliques dynamically fail to synergistically support the ipsi-lateral hip abductors to hold the contra-lateral hip in equilibrium, producing Trendelenburg gait. These contra-lateral external obliques have become weakened and lengthened due to an anteriorly tilted pelvis. These anatomical and biomechanical factors must be evaluated by the biokineticists/exercise therapists before prescribing a rehabilitative programme to ensure successful rehabilitation of lumbo-pelvic hip complex.

\section{Conflicts of Interest}

The authors declare no conflicts of interest regarding the publication of this paper.

\section{References}

[1] Castillo, E.R. and Lieberman, D.E. (2016) Lower Back Pain. Evolution, Medicine and Public Health, 1, 2-3.

[2] Kamal, Y. (2015) Lower Back Pain: Evolution of Back School Therapy. Journal of Spine, 4, 238. https://doi.org/10.4172/2165-7939.1000238

[3] Astfalck, R.G., O’Sulliva, P.B., Strakes, L.M., Smith, A.J., Burnett, A., Canceiro, J.P. and Dankaerts, W. (2010) Sitting Postures and Trunk Muscle Activity in Adolescents with and without No-Specific Chronic Low Back Pain: An Analysis Based on Sub-Classification. Journal of Spine, 35, 1387-1395. https://doi.org/10.1097/BRS.0b013e3181bd3ea6

[4] Laird, R.A., Gilbert, J., Kent, P. and Keating, J.L. (2014) Comparing Lumbo-Pelvic Kinematics in People with and without Back Pain: A Systematic Review and Meta-Analysis. BMC Musculoskeletal Disorders, 10, 299. https://doi.org/10.1186/1471-2474-15-229

[5] Deyo, R.A. and Weinstein, J.N. (2001) Low Back Pain. New England Journal of Medicine, 344, 363-370. https://doi.org/10.1056/NEJM200102013440508

[6] Hoy, D., Brooks, P. and Blyth, F. (2010) The Epidemiology of Low Back Pain. Best Practise Research in Clinical Rheumatology, 24, 769-781. https://doi.org/10.1016/j.berh.2010.10.002

[7] Nourbakhsh, M.R. and Arab, A.M. (2002) Relationship between Mechanical Factors and Incidence of Low Back Pain. Journal of Orthopaedic Sports Physical Therapy, 32, 447-460. https://doi.org/10.2519/jospt.2002.32.9.447

[8] O’Sullivan, P.B., Mitchell, T., Bulich, P., Waller, R. and Holte', J. (2006) The Relationship between Posture and Back Muscle Endurance in Industrial Workers with Flexion Related Low Back Pain. Manual Therapy, 11, 264-271. https://doi.org/10.1016/j.math.2005.04.004

[9] Brukner, P. and Khan, K. (2006) Clinical Sports Medicine. 3rd Edition, The McGraw Hill Company, New York.

[10] Gotlin, R.S. (2008) Sports Injuries Guidebook. Human Kinetics, Champaign, IL.

[11] Houglum, P.A. (2005) Therapeutic Exercises for Musculoskeletal Injuries. 2nd Edition, Human Kinetics, Champaign, IL.

[12] Kendall, F.P., McCreary, E.K., Provance, P.G., Rogers, M.M. and Romani, W.A. (2005) Muscles Testing and Function with Posture and Pain. Lippincott Williams \& Wilkins, Philadelphia, USA. 
[13] Prentice, W.E. (2011) Rehabilitation Techniques for Sports Medicine and Athletic Training. 5th Edition, Human Kinetics, Champaign, IL.

[14] Mansfield, P.J. and Neumann, D.A. (2009) Essentials of Kinesiology for the Physical Therapist Assistant. Mosby, Inc., St. Louis, Missouri, USA.

[15] Fuss, F.K. and Bacher, A. (1991) New Aspects of the Morphology and Function of the Human Hip Joint Ligaments. American Journal of Anatomy, 192, 1-13. https://doi.org/10.1002/aja.1001920102

[16] De Koning, C., Miller, M., Tucker, C. and Ellapen, T.J. (2014) Does an Anteriorly Tilted Pelvis Adversely Influence the Elderly's Ability to Sit to Stand? African Journal of Physical Health Education Recreation and Dance, 20, 646-654.

[17] Ellapen, T.J., Narsigan, S., Abrahams, S. and Desai, F.A. (2014) The Association of Unsound Sitting Posture and Vertebral Musculoskeletal Pain among University Administrators. Journal of Ergonomics, S4, 001. https://doi.org/10.4172/2165-7556

[18] Vidyasankar, V., Pherwani, A.D. and Hannon, R. (2003) Injuries to Abdomen and Pelvis. Surgery (Oxford), 21, 185-189. https://doi.org/10.1383/surg.21.8.185.16422

[19] Lee, J.H., Yoo, W.G. and Gak, H.B. (2011) The Immediate Effect of Anterior Pelvic Tilt Taping on Pelvic Inclination. Journal of Physical Therapy Science, 23, 201-203. https://doi.org/10.1589/jpts.23.201

[20] Baratta, R., Solomonow, M., Zhou, B.H., Letson, D., Chuinard, R. and D’Amborosia, R. (1988) Muscular Co-Activation: The Role of the Antagonist Musculature in Maintaining Knee Stability. American Journal of Sports Medicine, 16, 113-122. https://doi.org/10.1177/036354658801600205

[21] Paul, Y., Swanepoel, M., Ellapen, T.J., Muller, R.W. and Williams, J. (2016) What Biomechanical Value Can Athletes Gain from Symmetrically Strengthening Their Hip Extensors?

[22] Levangie, P. and Norkin, I. (2011) Joint Structure and Function: A Comprehensive Analysis. 4th Edition, E.A. Davis, Philadelphia.

[23] Speck, J. (2016). https://www.google.co.za/search?q=anteriorpelvictilt

[24] Stastny, P., Tufano, J.J., Lehnert, M., Golas, A., Zatar, A., Xaverova, Z. and Masczyk, A. (2015) Hip Abductors and Thigh Muscles Strength Ration and Their Relation to Electromyography Amplitude during Split Squat and Walking Lunge Exercises. Acta Gymnica, 45, 51-59. https://doi.org/10.5507/ag.2015.011

[25] Marieb, E. (2005) Human Anatomy and Physiology. 5th Edition, Human Kinetics, Champaign.

[26] Tyson, A.D. (2000) The Hip Flexor? Role in Pelvic Stability during Abdominal Exercises. Journal of Strength and Conditioning, 22, 57-57. 